This paper was published in Optics Letters and is made available as an electronic reprint with the permission of OSA. The paper can be found at the following URL on the OSA website: http://www.opticsinfobase.org/abstract.cfm?URI=ol-22-8-567. Systematic or multiple reproduction or distribution to multiple locations via electronic or other means is prohibited and is subject to penalties under law. 


\title{
pH sensor using Langmuir-Blodgett overlays on polished optical fibers
}

\author{
D. Flannery \\ Optical Sensors Group, School of Mechanical Engineering, Centre for Molecular Electronics and Centre for Photonics and
} Optical Engineering, Cranfield University, Cranfield, Bedford MK43 OAL, UK

\section{S. W. James and R. P. Tatam}

Optical Sensors Group, Centre for Photonics and Optical Engineering, School of Mechanical Engineering, Cranfield University, Cranfield, Bedford MK43 OAL, UK

\section{G. J. Ashwell}

Centre for Molecular Electronics and Centre for Photonics and Optical Engineering, Cranfield University, Cranfield, Bedford MK43 OAL, UK

Received November 18, 1996

Evanescent coupling between a side-polished single-mode optical fiber and a single-mode, $\mathrm{pH}$-sensitive Langmuir-Blodgett overlay is used to demonstrate an intrinsic fiber-optic $\mathrm{pH}$ sensor. The sensor shows a wavelength sensitivity of $18.8 \pm 0.8 \mathrm{~nm} / \mathrm{pH}$ and a transmission sensitivity of $9.7 \pm 0.8 \mathrm{~dB} / \mathrm{pH}$ when operating at $750 \mathrm{~nm}$. 1997 Optical Society of America

Fiber-optic chemical sensors offer several advantages over conventional chemical-sensing systems, ${ }^{1}$ specifically immunity to electromagnetic interference, the possibility of distributed sensing over long lengths of fiber, and, because of the guided nature of the light, their capability for safe operation in hazardous environments.

Intrinsic evanescent-wave fiber-optic chemical sensors have included absorption-based sensors, ${ }^{2}$ in which the evanescent field of the guided light is absorbed by the chemical of interest. This requires that the chemical being sensed have an absorption band that overlaps the wavelength of existing laser sources, thus limiting the application of this technique to particular chemical species. One way of overcoming this is to coat the optical fiber with a chemically sensitive reagent, which then exhibits a change in one of its optical properties, such as fluorescence ${ }^{3}$ when exposed to the chemical of interest. This change can then be addressed with sources of other wavelengths. As the light in evanescent-wave sensors is in close proximity to the chemical species of interest, these sensors exhibit fast response times. However, as only the optical power in the evanescent field is used, the sensitivity of such sensors can be limited. The sensor proposed in this Letter overcomes this problem by coupling the optical power from the fiber core into the chemically sensitive overlay, thus greatly increasing the optical power within the coating and maximizing the sensitivity of the sensor while retaining a fast response time.

In-fiber, channel-dropping filters that use a sidepolished, single-mode optical fiber, evanescently coupled to a single-mode Langmuir-Blodgett (LB) overlay waveguide ${ }^{4,5}$ exhibit a wavelength response that is highly dependent on the thickness and refractive index of the LB film. Intrinsic fiber-optic chemical sensors can be made by use of LB films that

0146-9592/97/080567-03\$10.00/0 change their optical characteristics when exposed to a particular chemical species.

Fiber-optic channel-dropping filters consist of a sidepolished, single-mode optical fiber onto which a material of higher refractive index $\left(n_{0}\right)$ than the fiber core $\left(n_{c}\right)$ is directly deposited (Fig. 1). A coupledwaveguide structure is formed when a film of the correct thickness is deposited onto the polished fiber. Optical power is evanescently coupled from the fiber core into the planar overlay at the wavelength at which the two waveguides are phase matched. The phasematching wavelength, $\lambda_{m}$, depends on the thickness and refractive index of the overlay, together with the polishing depth. One can calculate this by inserting the phase-matching condition into the eigenvalue equation for the zeroth-order mode of a three-layer asymmetric isotropic slab waveguide ${ }^{6}$ :

$$
\frac{2 \pi d}{\lambda_{m}} \sqrt{n_{0}^{2}-n_{\mathrm{eff}^{2}}}=\Phi_{s}+\Phi_{\mathrm{cl}},
$$

where $n_{\text {eff }}$ is the effective refractive index of the fiber mode, $d$ is the thickness of the overlay, and $\Phi_{s}$ and $\Phi_{\mathrm{cl}}$ are polarization-dependent phase terms for the superstate and cladding boundaries, respectively.

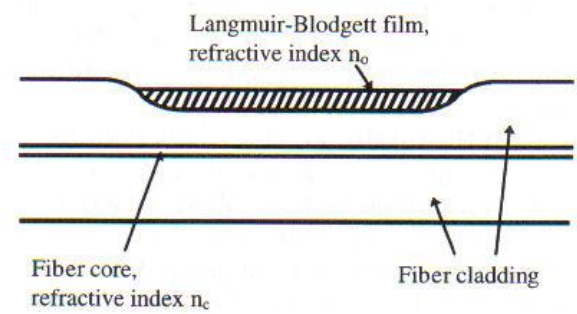

Fig. 1. Schematic diagram of the polished-fiber channeldropping filter.

(c) 1997 Optical Society of America 


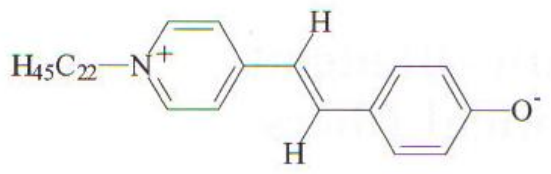

Fig. 2. Molecular structure of the merocyanine dye.

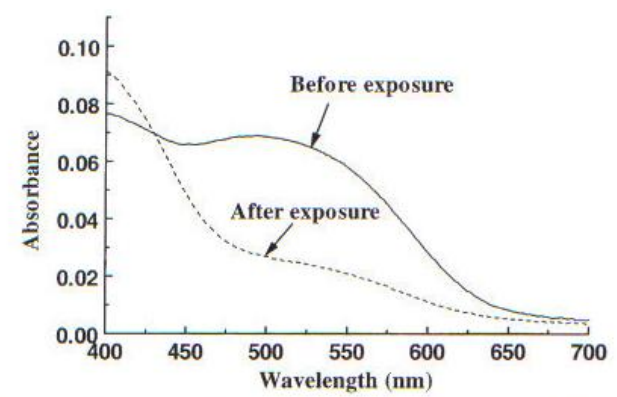

Fig. 3. Absorption spectra of 20 LB layers of the merocyanine dye, deposited onto a glass slide, before and after exposure to $\mathrm{HCl}$ vapor.

The resonant wavelength, $\lambda_{m}$, is particularly sensitive to the overlay thickness ${ }^{7}$ and therefore LB deposition ${ }^{8}$ is ideal because it allows organic materials to be deposited one molecular layer $(\sim 3 \mathrm{~nm})$ at a time and results in extremely accurate control of the overlay thickness.

A merocyanine dye, $E$ - $N$-docosyl-4-[2-(4-oxyphenyl) ethenyl] pyridinium inner salt (Fig. 2), was chosen as the overlay material because its terminal oxygen atom can be reversibly protonated. The dye was spread from chloroform $\left(0.15 \mathrm{mg} \mathrm{mL}^{-1}\right)$ onto the pure water subphase (Mili-Q, resistivity $18 \mathrm{M} \Omega \mathrm{cm}^{-1}$ at $25^{\circ} \mathrm{C}$ ) at a $\mathrm{pH}$ of 5.5 of a Nima Technology LB trough. After $10 \mathrm{~min}$ the surface layer was compressed at a rate of $30 \mathrm{~cm}^{2} \mathrm{~s}^{-1}$. Films were deposited onto a glass microscope slide, on the upstroke, at a surface pressure of $36 \mathrm{mN} \mathrm{m}^{-1}$. Six layers were deposited at a speed of $3 \mathrm{~mm} \mathrm{~min}^{-1}$ and a further 14 at $30 \mathrm{~mm} \mathrm{~min}^{-1}$. Absorption spectra were obtained with a Perkin-Elmer Lambda UV/VIS spectrophotometer before and after exposure to concentrated $\mathrm{HCl}$ vapor (Fig. 3). The dye predictably exhibited a large change in its absorption when exposed to the acid, and this resulted in a change in its refractive index at a given wavelength. Furthermore, this change was observed to be reversible when the slide was exposed to ammonia solution.

A side-polished optical fiber was prepared with a single-mode optical fiber (Spectran SMC-A0630B, cutoff wavelength $\sim 580 \mathrm{~nm}$ ), using an annular polishing technique. ${ }^{5}$ The polishing depth of the fiber was assessed with the oil-drop test. ${ }^{9}$ This resulted in $25 \%$ of the light being coupled out of the fiber when oil ( $n=1.464)$ was added to the polished region over an interaction length of approximately $2 \mathrm{~mm}$.

The merocyanine dye was deposited onto the sidepolished optical fiber with the parameters previously discussed. The channel-dropping response was monitored during deposition with the experimental setup shown in Fig. 4. Light from a white-light source was passed through a monochromator and then launched into a length of single-mode optical fiber by a polarizer and a $\times 20$ microscope objective. The state-of-polarization controller was used to define the polarization of the light in the device. The output fiber was retrieved from the second compartment of the LB trough so as to avoid disruption of floating monolayer and the transmitted light monitored by a photodiode and lock-in amplifier. A 56-layer LB film was deposited onto the polished fiber, forming a single-mode overlay waveguide, resulting in a channeldropping response centered at $725 \mathrm{~nm}$.

We tested the sensor by removing it from the LB trough and placing it in a temperature-controlled test cell; $7 \mathrm{~mL}$ of each solution of known $\mathrm{pH}$ was introduced into the test cell and allowed to reach a constant $25^{\circ} \mathrm{C}$. The monochromator was scanned through a set wavelength range, and the response of the sensor was recorded with the lock-in amplifier. This response was normalized to the emission spectrum of the whitelight source and fiber characteristics (Fig. 5).

The sensor can be interrogated by two different methods. The first is a wavelength-based method in which the resonant wavelength of the sensor gives a direct measure of $\mathrm{pH}$. This method requires no

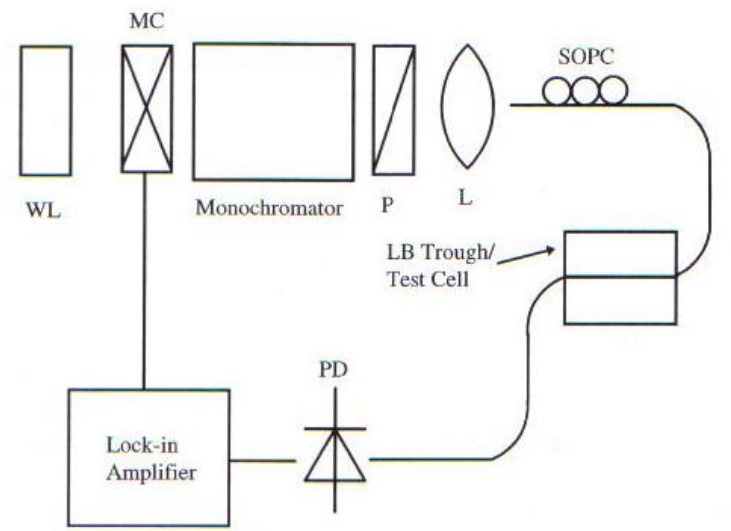

Fig. 4. Experimental apparatus: WL, white-light source; MC, mechanical chopper; P, polarizer; L, $\times 20$ microscope objective; SOPC, state-of-polarization controller; $\mathrm{PD}$, photodiode.

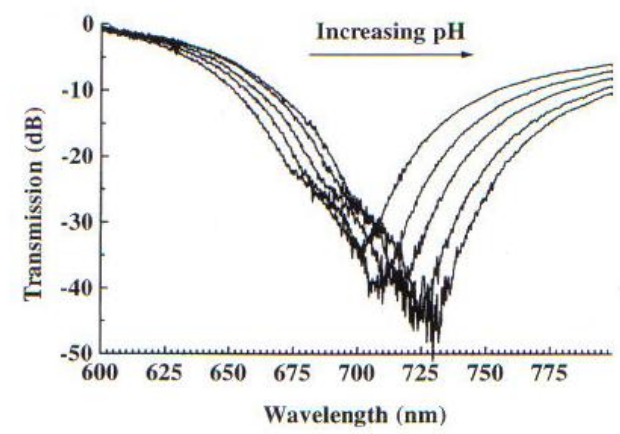

Fig. 5. Normalized TM transmission of the sensor: from left to right, the curves are for $11.52,11.99,12.31,12.67$, and $13.16 \mathrm{pH}$. 


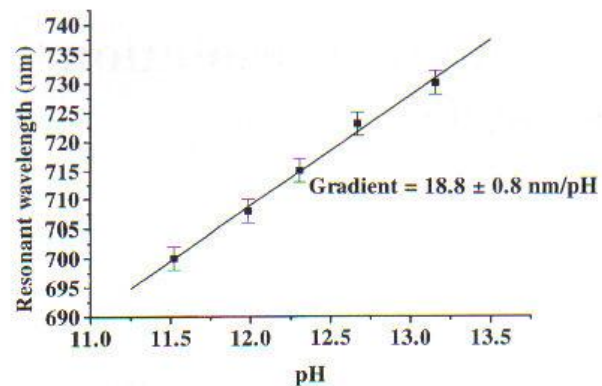

Fig. 6. Plot of resonant wavelength versus solution $\mathrm{pH}$.

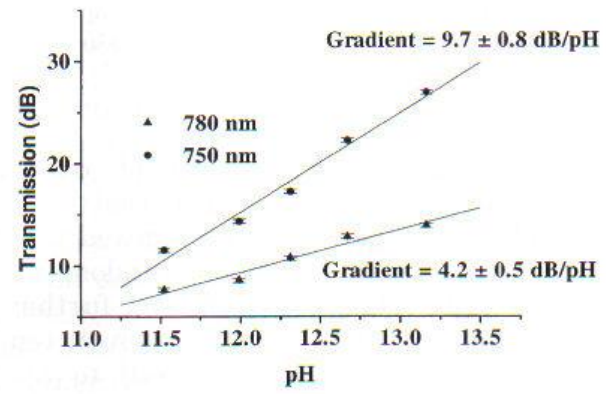

Fig. 7. Plot of sensor transmission versus solution $\mathrm{pH}$ for operating wavelengths 750 and $780 \mathrm{~nm}$.

intensity referencing. A plot of resonant wavelength versus $\mathrm{pH}$ is shown in Fig. 6, and linear regression analysis yields a linear response of $18.8 \pm 0.8 \mathrm{~nm} / \mathrm{pH}$ and a resolution of $0.1 \mathrm{pH}$. Second, an intensity-based method can be used. In this method the transmission of the sensor is measured at a single wavelength with a laser. From the results shown in Fig. 5 a plot of sensor transmission versus $\mathrm{pH}$ was obtained, as shown in Fig. 7. The sensitivity of this method is dependent on the laser wavelength, and to illustrate this, Fig. 7 shows the data for operating wavelengths of 750 and $780 \mathrm{~nm}$. Using a linear regression analysis, one can see that the sensor is considerably more sensitive when operated at $750 \mathrm{~nm}(9.7 \pm 0.8 \mathrm{~dB} / \mathrm{pH})$ than at $780 \mathrm{~nm}(4.2 \pm 0.5 \mathrm{~dB} / \mathrm{pH})$. Assuming temperature stabilization of $\pm 0.01^{\circ} \mathrm{C}$, we predict a resolution of $\sim 0.001 \mathrm{pH}$.

A response time of approximately $20 \mathrm{~s}$ was measured when the transmission of the sensor was monitored with a laser diode source operating at $780 \mathrm{~nm}$ while the solution $\mathrm{pH}$ was increased. A response time corresponding to lowering the solution $\mathrm{pH}$ was unachievable, as it was too short to allow measurements to be made,

The dynamic range of the sensor is limited by the thickness of the overlay waveguide. In this Letter the LB overlay was approximately $180 \mathrm{~nm}$ thick, and solutions of $\mathrm{pH} \leq 11$ saturated the response. By depositing thicker, multimode overlay waveguides, one should be able to increase the dynamic range to allow measurement of lower $\mathrm{pH}$ solutions, probably at the expense of response time.

One can readily extend the technique used to manufacture this $\mathrm{pH}$ sensor to fabricate sensors for other chemical species, for example, by choosing suitably sensitive materials, for the LB deposition. We are also investigating the possibility of surface plasmon resonance devices, where a thin layer of metal is deposited between the optical fiber cladding and the overlay. Initial numerical analysis of such sensors shows that this should result in a narrowing of the linewidth and an increased sensitivity.

D. Flannery thanks the UK Engineering and Physical Sciences Research Council for a studentship.

\section{References}

1. R. A. Lieberman, Sensors Actuators B 11, 43 (1993).

2. H. Tai, H. Tanaka, and T. Yoshino, Opt. Lett. 12, 437 (1987).

3. B. D. MacCraith, V. Ruddy, and C. Potter, Electron. Lett. 27, 1247 (1991)

4. R. B. Charters, A. P. Kuczynski, S. E. Staines, R. P. Tatam, and G. J. Ashwell, Electron. Lett. 30, 594 (1994).

5. R. B. Charters, S. E. Staines, and R. P. Tatam, Opt. Lett. 19, 2036 (1994).

6. V. Ramaswamy, Appl. Opt. 13, 1363 (1974).

7. M. Zhang and E. Garmire, J. Lightwave Technol. 5, 260 (1987).

8. I. R. Peterson, J. Phys. D. 23, 379 (1990).

9. O. G. Leminger and R. Zengerle, J. Lightwave Technol. 3, 864 (1985). 